\title{
Ammesso e non concesso che si possa usare anche dato e non concesso
}

Luisa di Valvasone

PUBBLICATO: 18 GIUGNO 2019

\section{Quesito:}

Diversi lettori ci scrivono in merito alla locuzione ammesso e non concesso, il cui significato rimane talvolta opaco. Altri, come Giovanni P., si domandano se non sia più corretta l'espressione dato e non concesso e se le due locuzioni abbiano lo stesso significato.

\section{Ammesso e non concesso che si possa usare anche dato e non concesso}

1 mmesso e non concesso (che) è una locuzione congiuntiva (ovvero una sequenza di parole che nell'insieme svolge le funzioni di una congiunzione) che introduce una proposizione concessiva-ipotetica con il verbo di norma al congiuntivo (raramente all'indicativo nell'uso informale), anche se non è insolito trovare usi assoluti del tipo ammessa e non concessa la tua innocenza, potevi comunque comportarti meglio. Per quanto riguarda il significato, con questa espressione chi parla o chi scrive vuole mettere una certa distanza tra sé e ciò che è ammesso e non concesso. Secondo il Devoto-Oli 2019 la locuzione "introduce uno o più elementi che si ritiene di dover mantenere per il momento al livello di semplice ipotesi: ammesso e non concesso che si trattasse di un errore"; per il GRADIT è usata "per indicare che si ammette momentaneamente come vera un'ipotesi ritenuta improbabile: ammesso e non concesso che sia come tu dici, non c'era bisogno di reagire cosi"; il Vocabolario Treccani online aggiunge, intensificando la "presa di distanza" da quanto ipotizzato, che si usa "quando si ammette momentaneamente come vera un'ipotesi ritenuta improbabile, al solo scopo di poter controbattere le ragioni dell'avversario o per aver modo di sviluppare un ragionamento: ammesso e non concesso che ti abbia offeso, non avevi diritto di reagire cosi".

In merito invece all'espressione dato e non concesso, che alcuni lettori propongono come alternativa più corretta rispetto ad ammesso e non concesso, va subito precisato che i dizionari sincronici consultati (Devoto-Oli 2019, Zingarelli 2019, Vocabolario Treccani, Sabatini-Coletti 2008, GRADIT) trattano le due locuzioni come sinonimiche e alternabili nell'uso, e le definizioni rimandano spesso l'una all'altra; soltanto il Vocabolario Treccani, alla voce concedere, segnala dato e non concesso come "meno comune". Nel dizionario storico GDLI, alla voce concesso, troviamo: "Dato (o ammesso) e non concesso: nelle discussioni, quando si accoglie momentaneamente come vera un'ipotesi ritenuta improbabile, al fine di meglio controbattere le argomentazioni di altri o sviluppare le proprie" a cui seguono attestazioni prese da Sassetti, Borghini e Galileo Galilei, tutte di dato e non concesso (o con la forma antica del participio passato conceduto). Il Tommaseo-Bellini e la quinta edizione del Vocabolario degli Accademici della Crusca (la locuzione è assente nelle prime quattro impressioni), invece, riportano solo la forma dato e non concesso. Riportiamo la definizione, che si trova alla voce conceduto nella quinta Crusca, corredata dagli esempi tratti dal commediografo cinquecentesco Raffaello Borghini (ma questo esempio è già presente nella quarta impressione, al lemma adducere), da Galileo Galilei e dal poema eroicomico settecentesco La Celidora di Andrea Casotti:

Dato e non concesso che, o Data e non concessa la tal cosa, è maniera con la quale, pur negando la sostanza della proposizione dell'avversario, si vuol far intendere che, anche ammessa, sarebbe sempre vero ciò che da noi è sostenuto- 
Borgh. R. Rip. 22 [Raffaello Borghini, Il riposo]: Dato e non conceduto che questa ragione potesse adducere. Galil. Op. astronom. 1, 74 [Galileo Galilei, Operazioni Astronomiche]: Dato e non concesso che nella luna fusse chi di là potesse rimirar la terra, vedrebbe ogni giorno tutta la superficie terrestre. Casott. A. Celid. 3,52 [Andrea Casotti, La Celidora]: E dato e non concesso senza freno Che s'empiano [i topi], è poi lor tanto veleno.

Le due espressioni si formano dall'unione delle distinte locuzioni ammesso che/dato che e concesso che costruite rispettivamente dai participi passati dei verbi ammettere, dare e concedere + che - le quali possono avere nell'uso comune significati equivalenti. Nella Grammatica italiana di Luca Serianni (Serianni 1989) ammesso che, dato che e concesso che sono tutte identificate come locuzioni congiuntive, a cui segue di norma il congiuntivo (dato che + indicativo introduce invece una proposizione causale), che «possono assumere valore concessivo, specie se accompagnate da anche o pure: "anche ammesso che tu avessi ragione, hai fatto male a comportarti cosi (= anche se avevi, pur avendo)"» (p. 596). Da queste nascono le locuzioni dato e non concesso (che) e ammesso e non concesso (che) che convivono da tempo nell'italiano condividendo lo stesso significato, come si può verificare dagli esempi riportati sotto, tratti da differenti autori e con diverse datazioni, nei quali la scelta dell'una o dell'altra forma dipende di volta in volta dal gusto dello scrittore (volendo fare un piccolo esperimento, se sostituissimo le locuzioni l'una con l'altra il significato resterebbe lo stesso):

Dato e non concesso che mi scriva, dimmi se le società (dette altrimenti accademie) di casa Sartori e Mazio sono sempre sull'istesso piede (Massimo D’Azeglio, Epistolario (1819-1866), a cura di G. Virlogeux, voll. I-V, Torino, 1987-2002).

Del resto, ammesso e non concesso che io allunghi l'atto operativo; ma è proprio vero che il compenso proposto dal Cav. Egidi col suo dilatatore bivalve, abbrevia la operazione? (Bollettino delle malattie dell'orecchio, della gola e del naso, anno VII, Firenze, I889).

Dal punto di vista poetico (dato e non concesso che di poesia possa parlarsi) si tratterebbe dunque di una poesia che mette insieme pezzi prefabbricati. Operazione che, dopo Laforgue, è stata spesso tentata da poeti cubisti e surrealisti (Eugenio Montale, Il quasi-melodramma del vecchio maestro, "Il Corriere della Sera", 1966).

«Salvo, quelle foto, ammesso e non concesso che ottieni [sic] il permesso di raprire [sic] la cassetta, ammesso e non concesso che le foto siano ancora là dintra, ammesso e non concesso che il magistrato te le lassi in mano per più di dù secondi, non rappresentano 'na minchia di nienti!» (Andrea Camilleri, Le indagini di Montalbano, Sellerio Editore, Palermo, 1994).

Il DELI indica come data di prima attestazione per dato e non concesso il 1573 (in uno scritto di Filippo Sassetti: "Io credo che costui non abbia saputo dir male con fondamento, dato e non concesso (come dice lui) che e' dica il vero in dimolte cose."), mentre per ammesso e non concesso troviamo sul corpus di Google Libri un'attestazione risalente al I6o3:

[...] conciosia che per la profondità della dottrina sua meritò esser detto Segretario della Natura. Ma ammesso, e non concesso tutto questo, deono nondimeno avertire, che colui sarebbe del nome di segretario indegno, che temerariamente i segreti fidatigli dal suo Principe palesasse (Cesare della Riviera, Il magico mondo degli heroi, Francesco Osanna, Mantova, I603).

Nell'uso comune attuale sembrerebbe esservi una netta prevalenza per ammesso e non concesso: sulle pagine in italiano di Google (al 25/2/2019) si registrano I70.0oo risultati per ammesso e non concesso contro ir6.roo per dato e non concesso; all'incirca le stesse proporzioni si ritrovano negli archivi dei quotidiani (archivio della "Repubblica": 765 risultati per ammesso e non concesso a partire dal I984 e29 
risultati per dato e non concesso a partire dal 1987; archivio del "Corriere": 6oo risultati per ammesso e non concesso a partire dal 1889 , di cui 265 dal 2000 a oggi, e 367 risultati per dato e non concesso a partire dal I876, di cui solo 5 dal 2000 a oggi); e anche la ricerca sul CORIS - corpus di italiano scritto che raccoglie testi di diverse tipologie (stampa, saggistica, narrativa) degli anni Ottanta e Novanta conferma i risultati già visti con 22 occorrenze per ammesso e non concessoe solo 2 per dato e non concesso.

Se questi dati confermano la prevalenza d'uso nell'italiano contemporaneo della forma ammesso e non concesso, l'analisi più approfondita e il confronto con i risultati ricavati dalla ricerca sul corpus di Google Libri ci mostrano, almeno nell'uso letterario, un cambio di tendenza avvenuto presumibilmente nel corso del Novecento. Infatti, il totale dei risultati che emerge dalle pagine in italiano di Google Libri (il 25/2/2019) ribalta la situazione vista finora: circa 13.400 risultati per ammesso e non concesso (a cui si possono sommare le 5 occorrenze per la forma arcaica ammesso e non conceduto) e circa 22.600 risultatiper dato $e$ non concesso (e r.210 per dato $e$ non conceduto,prevalentemente sette e ottocenteschi). Guardando al totale delle occorrenze spicca dunque una netta prevalenza per dato e non concesso, ma analizzando i singoli risultati si nota che la maggior parte delle occorrenze di questa forma risale al XX secolo (I3.30o) e al XIX secolo (6.5Io) e diminuisce vistosamente nel XXI secolo, mentre le occorrenze diammesso e non concesso aumentano proporzionalmente nel XX e nel XXI secolo (si noti inoltre che non risultano su Google Libri attestazioni settecentesche per ammesso e non concesso); la tabella seguente mostra i dati approssimativi:

\begin{tabular}{|l|l|l|l|l|}
\hline & XIX secolo & XX secolo & XXI secolo & $\begin{array}{l}\text { Totale } \\
\text { occorrenze }\end{array}$ \\
\hline $\begin{array}{l}\text { Ammesso e } \\
\text { non concesso }\end{array}$ & $\begin{array}{l}1.120 \\
(8 \%)\end{array}$ & $\begin{array}{l}5.990 \\
(45 \%)\end{array}$ & $\begin{array}{l}6.250 \\
(47 \%)\end{array}$ & $\mathbf{1 3 . 4 0 0}$ \\
\hline $\begin{array}{l}\text { Dato e non } \\
\text { concesso }\end{array}$ & $\begin{array}{l}6.510 \\
(29 \%)\end{array}$ & $\begin{array}{l}13.300 \\
(59 \%)\end{array}$ & $\begin{array}{l}2.640 \\
(12 \%)\end{array}$ & $\mathbf{2 2 . 6 0 0}$ \\
\hline
\end{tabular}

In questo caso sono utili anche i dati ricavati dalle ricerche sul DIACORIS (corpus d'italiano che prende in considerazione differenti tipologie di testi a partire dal I86I fino al 200I), in cui si trovano 4 risultati di ammesso e non concesso e 6 risultati di dato e non concesso, e sulla BIZ, nella quale vi è un'unica occorrenza per ammesso e non concesso (nella novella pirandelliana "Donna Mimma" del ig25), ma 8 occorrenze per dato e non concesso (da Galilei a Pirandello, si noti l'alternanza d'uso nello stesso autore) e una per dato e non conceduto (nel trattato La Supplica di N. Barbieri, I634). L'inversione di tendenza verificatasi a cavallo tra il XX e il XXI secolo è infine ben visibile anche dal grafico che restituisce Google NGram ricercando le nostre due espressioni a partire dal I8oo: 


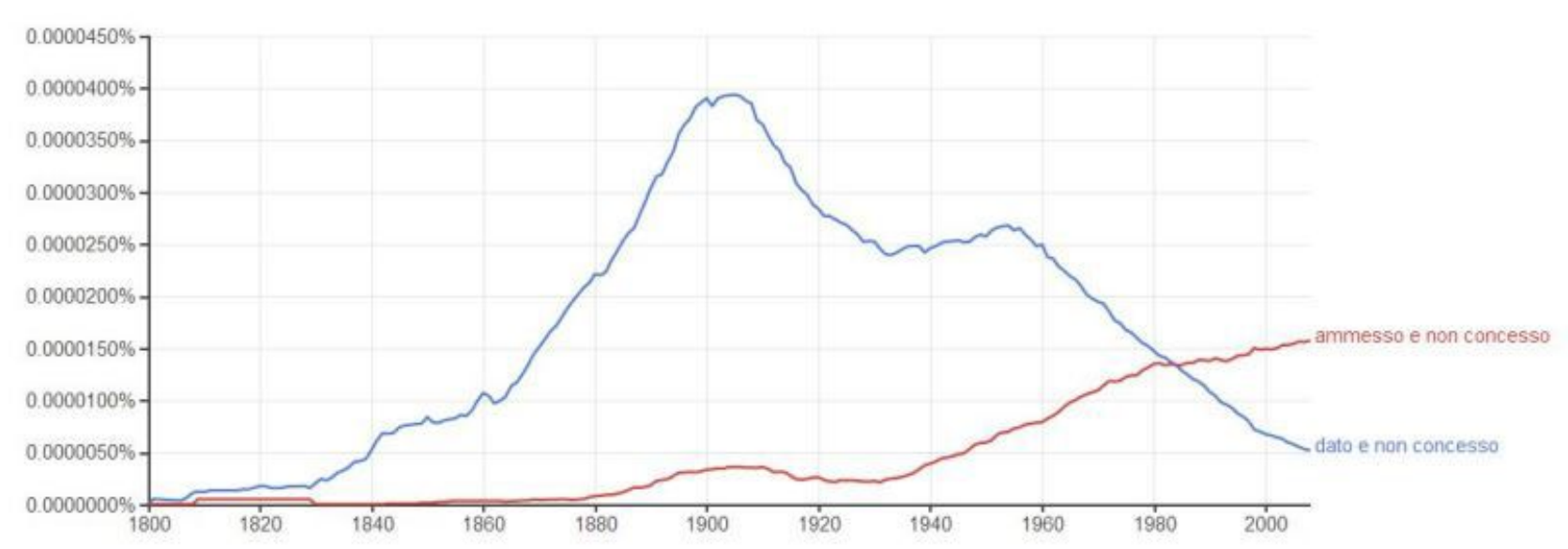

Tirando le somme, sembrerebbe che dato e non concesso (o conceduto) abbia goduto di maggior prestigio fino alla metà del XX secolo (ed è forse questo uno dei motivi che potrebbe spingere alcuni lettori a percepirlo come più corretto rispetto ad ammesso e non concesso) per poi ritirarsi gradualmente in favore della forma, oggi predominante, ammesso e non concesso. Al successo di quest'ultima inoltre potrebbe aver contribuito nientemeno che il "principe della risata" Totò, padre di immortali modi di dire, che nel suo variegato linguaggio annovera anche la nostra locuzione; un esempio è il breve estratto che segue, tratto dallo sketch "Pasquale" di Totò e Mario Castellani, ospiti nel ig66 della trasmissione Rai Studio Uno:

Totò: Ora ti racconto un esipodio che mi è capitato stamattina.

Castellani: Non capisco cosa sarebbe questo esipodio.

Totò (deciso, scandendo le lettere): Un Esipodio.

Castellani: Ah, episodio.

Totò: Adesso si dice cosi.

Castellani: Da quando c'è la lingua italiana si dice cosi.

Totò: Ammesso e non concesso.

Castellani: Allora racconta.

Per rispondere infine ai dubbi dei lettori, nonostante l'attuale prevalenza d'uso di ammesso e non concesso rispetto all'ormai più letterario dato e non concesso, entrambe le espressioni sono corrette e la scelta dell'una o dell'altra dipende esclusivamente dallo stile e dal gusto di chi scrive o parla.

\section{Cita come:}

Luisa di Valvasone, Ammesso e non concesso che si possa usare anche dato e non concesso, "Italiano digitale", 2019, IX, 2019/2 (aprile-giugno)

DOI: $10.35948 / 2532-9006 / 2020.3125$

Copyright 2019 Accademia della Crusca

Pubblicato con licenza creative commons CC BY-NC-ND 